\title{
Adenoid Cystic Carcinoma of Trachea
}

\author{
Ashish Varghese $^{1} \cdot$ Swati Suneha $^{1} \cdot$ John C. Watkinson ${ }^{2}$
}

Received: 21 September 2015 / Accepted: 10 February 2016 / Published online: 13 February 2016

(C) Association of Surgeons of India 2016

\begin{abstract}
Adenoid cystic carcinoma (ACC) is the second most common primary malignant tumor of the trachea, after squamous cell carcinoma. Patients present with upper airway obstructive symptoms and signs including dyspnoea, cough, haemoptysis, and stridor which are often insidious, delaying diagnosis and optimal management. Described here is an unusual case of primary ACC cervical trachea with concomitant micropapillary thyroid carcinoma (microPTC) in a middleaged lady presenting with cough and breathing difficulty since 3 months.
\end{abstract}

Keywords Adenoid cystic carcinoma $\cdot$ Micro papillary thyroid carcinoma $\cdot$ Trachea $\cdot$ Stridor

\section{Introduction}

Primary tracheal tumors are extremely rare, accounting for approximately $0.2 \%$ of the entire respiratory system with 2.6 new cases per million people reported per year, and $80 \%$ of these are malignant $[1,2]$. Adenoid cystic carcinoma of the trachea is a type of adenocarcinoma arising from intercalated duct reserve cells of the terminal tubular complex,

Ashish Varghese

ashishvargheseent@yahoo.com

1 Otolaryngology-Head and Neck Surgery Department, Christian Medical College, Ludhiana 141008, Punjab, India

2 Queen Elizabeth University Teaching Hospital, Birmingham, UK which gives rise to tracheobronchial submucosal seromucinous glands [3] seen in middle age and has no association with smoking [4]. A predominantly submucosal growth, it is most commonly found in subglottis ( $65 \%$ ), followed by supraglottis (35\%) and rarely glottis. It is slow growing, locally aggressive with propensity for perineural invasion and haematogenous spread [3]. Surgical resection with negative margins and reconstruction is the preferred modality of treatment in view of its role in relieving airway obstruction followed by chemoradiotherapy.

\section{Case Description}

A 57-year-old hypertensive lady presented with complaints of nonproductive cough with progressive breathing difficulty for the past 3 months and worsening since the last 10 days. Orthopnoea was present; however, there was no fever, chest pain or history suggestive of laryngeal involvement. She was clinically euthyroid. There was no significant past or family history. A pulmonary function test (PFT) done outside had shown mild restrictive pattern, and the treadmill test (TMT) result was negative. She had taken symptomatic treatment from local alternative medicine practitioners and was also started on antihistaminics with no relief.

On clinical examination, she was morbidly obese with biphasic stridor. Her vital signs were within normal limits with no cervical or generalized lymphadenopathy. She maintained saturation without oxygen support. Cardiovascular and respiratory systems also were clinically normal. There was no thyroid gland enlargement or thyroid cartilage tenderness, and no cricothyroid escape and laryngeal crepitus was present. Laryngoscopy 
revealed a growth in the subglottic area with a marked airway compromise. However, bilateral vocal cords were symmetrically mobile and normal.

Her routine blood investigations and thyroid functions were within normal limits. CECT neck and upper chest showed diffuse enhancement, hypodensity involving isthmus, and left lobe thyroid with destruction of 1st, 2nd and probably 3rd cervical tracheal rings approximately $3.2 \mathrm{~cm}$ in length. There was marked soft tissue thickening around the rings resulting in airway narrowing. However, there was no retrosternal extension (Figs. 1 and 2). An ultrasound guided fine needle aspiration cytology (FNAC) was done which was suggestive of undifferentiated malignancy. An ultrasound abdomen revealed no evidence of metastasis. So a surgical resection was planned. A hard growth was visualized in the thyroid gland involving about 3-4 $\mathrm{cm}$ of tracheal rings. A frozen section was sent which revealed the growth to be adenoid cystic carcinoma, infiltrating the thyroid tissue. The patient then underwent total thyroidectomy with resection of $4 \mathrm{~cm}$ of cervical trachea and excision of the upper and lower tracheal rings for margin clearance. A laryngeal drop of approx $4 \mathrm{~cm}$ was achieved, and end-to-end tracheal anastomosis was done.

Biopsy was diagnostic of adenoid cystic carcinoma grade II of III, trachea with mixed cribriform and solid patterns. Tumor was found to infiltrate the thyroid gland. Superior and inferior margins were free of tumor. Lymphovascular and perineural invasion were present. There were metastatic carcinomatous deposits in paratracheal lymph nodes. Multicentric micropapillary carcinoma thyroid was incidentally identified. Patient was given post-operative chemoradiation with carboplatin and 54 Gy units of radiation. She is currently on regular follow-up with no evidence of recurrence or metastasis till date.

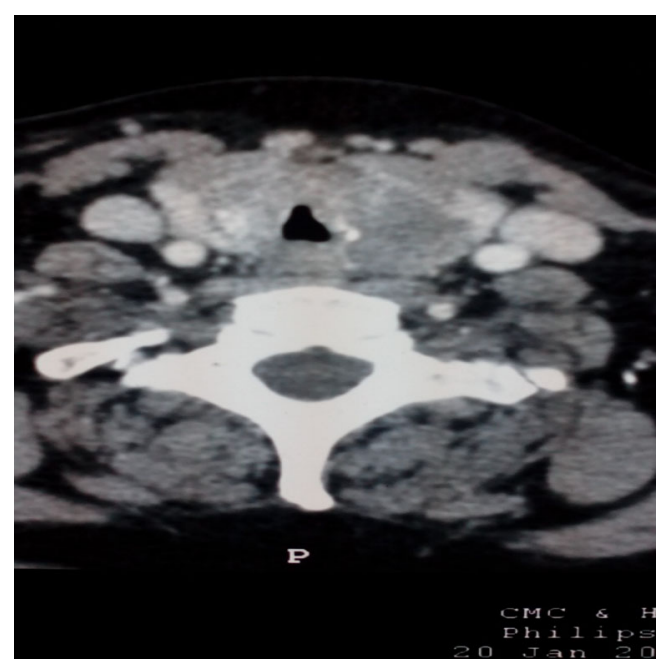

Fig. 1 Thyroid left lobe and isthmus hypodense and enhancing mass

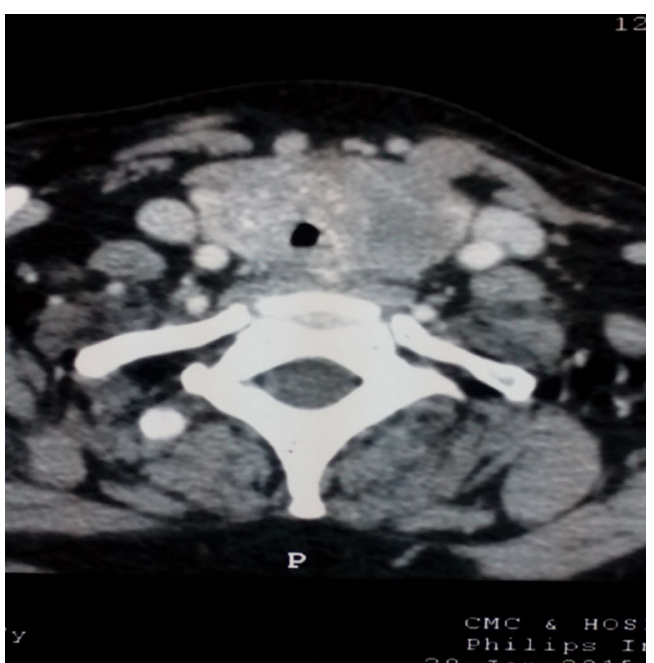

Fig. 2 Marked luminal compromise with tracheal ring destruction

\section{Discussion}

Adenoid cystic carcinoma (ACC) most commonly occurs in the salivary gland. In the trachea, it arises from the submucosal layer and has a rapid locoregional spread. ACC spreads most commonly by direct extension, submucosal, perineural invasion or hematogenous metastasis. Perineural spread makes surgical margin achievement a challenge. More than $50 \%$ of patients with tracheal ACC have hematogenous metastases, most common being pulmonary which can remain asymptomatic for many years. Metastases to the brain, bone, liver, kidney, skin, abdomen and heart have also been reported [4]. Positive nodes in the head and neck ACC are associated with decreased 10-year survival; however, its relevance in the survival of tracheal ACC is unclear. Patients present with insidious respiratory complaints of cough, wheezing, dyspnoea or haemoptysis. Provisional diagnosis of a tracheal tumor is reached with CT imaging features of tracheal wall thickening or luminal mass infiltrating trachea. Differentials include squamous cell carcinoma, carcinoid, mucoepidermoid, nonsquamous bronchogenic carcinoma, lymphoma and melanoma [5]. Final diagnosis of ACC is made only after histopathological confirmation [6]. Surgical resection is the mainstay of treatment as these patients almost always present with airway compromise, and thus, securing it becomes paramount. Tumor resection with end-to-end anastomosis achieved by laryngeal release and lower tracheal mobilization results in laryngeal preservation without compromising oncological margin [7]. Surgical complications include tracheoesophageal fistula, pharyngeal or oesophageal leak, anastomotic separation, wound dehiscence, vocal cord paralysis, temporary tracheotomy, dysphagia and pneumonia. Chemoradiation is given postoperatively with paclitaxel/carboplatin with good results. Palliative radiotherapy is also given in unresectable tumors or poor candidates for surgery [8]. 


\section{References}

1. Gaissert HA, Mark EJ (2006) Tracheobronchial gland tumors. Cancer Control 13(4):286-294

2. Urdaneta AI, Yu JB, Wilson LD (2011) Population based cancer registry analysis of primary tracheal carcinoma. Am J Clin Oncol 34(1):32-37

3. Armstrong WB, Vokes DE, Verma SP (2015) Malignant tumors of the larynx. In: Flint PW, Haughey BH, Lund VJ, Niparko JK, Robbins KT, Thomas JR et al (eds) Cummings otolaryngology, head and neck surgery, 6th edn. Elsevier Saunders, Philadelphia, pp 16011633

4. Yang PY, Liu MS, Chen CH, Lin CM, Tsao TC (2005) Adenoid cystic carcinoma of the trachea: a report of seven cases and literature review. Chang Gung Med J 28(5):357-363
5. Gaissert HA, Grillo HC, Shadmehr BM, Wright CD, Gokhale M, Wain JC et al (2005) Laryngotracheoplastic resection for primary tumors of the proximal airway. J Thorac Cardiovasc Surg 129(5): 1006-1009

6. Nomori H, Kaseda S, Kobayashi K, Ishihara T, Yanai N, Torikata C (1988) Adenoid cystic carcinoma of the trachea and main-stem bronchus. A clinical, histopathologic, and immunohistochemical study. J Thorac Cardiovasc Surg 96(2): 271-277

7. Subramaniam T, Lennon P, Kinsella J, O’Neill JP (2015) Laryngeal preservation in managing advanced tracheal adenoid cystic carcinoma. Case Rep Otolaryngol 2015:404586

8. Muller A, Stockamp B, Schnabel T (2000) Successful primary radiation therapy of adenoid cystic carcinoma of lung. Oncology $58: 15-17$ 